\title{
Mobile service aware opportunistic embedded architecture of mobile crowd sensing networks for power network measurement
}

\author{
Jianwei Zhang ${ }^{1,2^{*}}$ and Hao Yang ${ }^{1,2}$
}

\begin{abstract}
In order to improve the intelligent degree and robustness optimization of power grid management system, the opportunistic embedded architecture was proposed for power network measurement with mobile service aware scheme. First, the mobile crowd sensing network for power grid management was proposed to realize the intelligent power grid management. Then, we designed the mobile service aware opportunistic embedded system based on the requirements of intelligent power grid management and deployment of mobile crowd sensing network. Thirdly, the grid of embedded systems was demonstrated for intelligent management. The experimental results show that the proposed scheme has obvious advantages in system complexity, execution efficiency, intelligent power grid management level, etc.
\end{abstract}

Keywords: Opportunistic embedded architecture, Mobile crowd sensing, Power network measurement, Mobile service aware

\section{Introduction}

With the rapid development of the electricity grid, the wide application of intelligent electronic equipment brings great convenience and benefit for the human life and production at the same time; it also brings challenges to power grid management and reliable guarantee. However, unstable and inefficient power supply and power grid management will inevitably bring many difficulties to the human daily life and work. So, the effective management of mobile devices and smart grid management scheme and construction of reasonable, complete, and efficient smart grid management become the key.

With the help of a smart grid management platform, real-time monitoring, and the management of power use situation, a power monitoring system was designed based on a smart grid management platform [1]. Based on the existing power quality, a monitoring system of power quality management platform was proposed in

\footnotetext{
* Correspondence: zhangjianweijh@163.com

${ }^{1}$ Electric Power Research Institute of Yunnan Power Grid Co., Ltd, Kunming, China

${ }^{2}$ Key Laboratory of CSG for Electric Power Measurement, Kunming, China
}

article [2]. The authors of [3] proposed the integration service system of intelligent power grid management based on the Internet of Things service system. According to the generalized data automatic acquisition problem, the general data that can be used in the intelligent processing management model was studied [4]. Based on the limited capacity of Petri nets, a formal model of the smart grid transmission system was established in [5]. For large-scale automatic meteorological station network characteristics and requirements of data transmission, the special GPRS data communication terminals designed and the wireless data terminal hardware scheme are given by the authors of article [6]. A new scheme of high TCP availability was proposed in [7]. The program cycle structure was adjusted for optimizing and improving the local equipment's operation [8]. A set of power equipment monitoring system was designed based on the embedded system [9], which is mainly composed of ultraviolet probe, embedded computer system, and GPRS module. The aging test design scheme was proposed in [10]. However, the above articles 
ignored the opportunistic control characteristics of embedded devices.

A group of mental perception network constitutes that is people-centered was proposed based on the perception of social behavior analysis mode of the important way of data collection [11]. Mobile terminal group of mental perception mode was proposed in article [12]. Mobile group of city hot issues of data method was studied in [13], based on the hot issues for discovery and classification. A novel incentive mechanism based on multi-Interaction dynamic incentive mechanism was proposed in article [14]. [14]. A high level synthesis approach was presented in [15] for generating the embedded processor arrays for matrix algorithms based on the polytope model. A novel fingerprint template protection scheme based on chaotic encryption was proposed in [16]. The study of article [17] suggests that the near-tip conditions for both the left and right crack tips in systems with non-horizontal cracks are dominated by mixed mode conditions. A novel simple algorithm for dynamic intelligent load balancing was proposed for decreasing the power losses in a power distribution network [18]. An enhanced way-prediction cache was developed for coping with the weakness of the way-prediction cache [19]. Chutisant Kerdvibulvech proposed a methodology for motion analysis and hand tracking based on adaptive probabilistic models [20]. However, the above researches did not make the future research of the relationship between mobile crowd sensing networks and power network measurement.

On the basis of the above research results, the mobile service aware opportunistic embedded architecture of mobile crowd sensing networks for power network measurement automation was researched and presented.

The rest of the paper is organized as follows. Section 2 describes the mobile crowd sensing network for power grid management. In Section 3, we discussed the mobile service aware opportunistic embedded system. In Section 4, we proposed the grid intelligent management of the embedded systems. The performance analysis of embedded system has been shown in Section 5. Finally, the conclusions are given in Section 6.

\section{Mobile crowd sensing network for power grid management}

For realizing the intelligent power grid management, the following several issues should be considered:

(1) Grid embedded geographic information system

(2) Power grid management communication between the embedded devices

(3) Power grid management redundant components

(4) Grid embedded equipment maintenance management

(5) Grid communication optimization

(6) Embedded system operation and control
Here, the operation and control scheme of the embedded equipment of the intelligent power grid is the core of the intelligent network management. The core functionality can be subdivided the following several aspects:

(1) The embedded devices of power management

(2) The embedded devices working voltage intelligent control

(3) The self-protection function of embedded equipment

(4)Equipment group of cooperative control

(5) Feeder intelligent control of power grid

(6) Front-end perception data processing equipment

The above analysis shows that the grid management of intelligent electric power dispatching and intelligent substation of the crowd sensing network management could be realized through the deployment of embedded equipment and communication network and single front-end embedded devices of data perception, covering the area of intelligent network, real-time monitoring circuit load, and other important link in the management of power grid.

Therefore, in the power grid management, deployment of mobile crowd sensing network is shown in Fig. 1, including the embedded devices (ES) and subnet (SS). In urban, the suburban deploys embedded devices of different types and scale. The two-way communication link of the embedded devices, one of the Internet and mobile crowd sensing gateway and one of the gateway and the power grid management center, would be realized. Moreover, through the point-to-point bidirectional link, end-to-end communication between any two points would be realized as shown in Fig. 1.

In grid management front to join the embedded system, the function of the platform of the system is shown in Fig. 2. Additionally, Fig. 2 shows the four-step workflow of the embedded grid management.

Step 1. Embedded control equipment testing

Step 2. Embedded logic control

Step 3. Distributed ES point to point

Step 4. Connectivity of mobile crowd sensing networks

The above four steps and its features of the embedded devices and mobile group were established with the end-to-end communication between crowd sensing networks. Moreover, mobile crowd sensing networks could provide three aspects of the functions of grid data management for the embedded devices.

(1)Data collection. Embedded devices can sense the data, by collecting incentive mechanism and arousing enthusiasm of embedded devices data collection. 


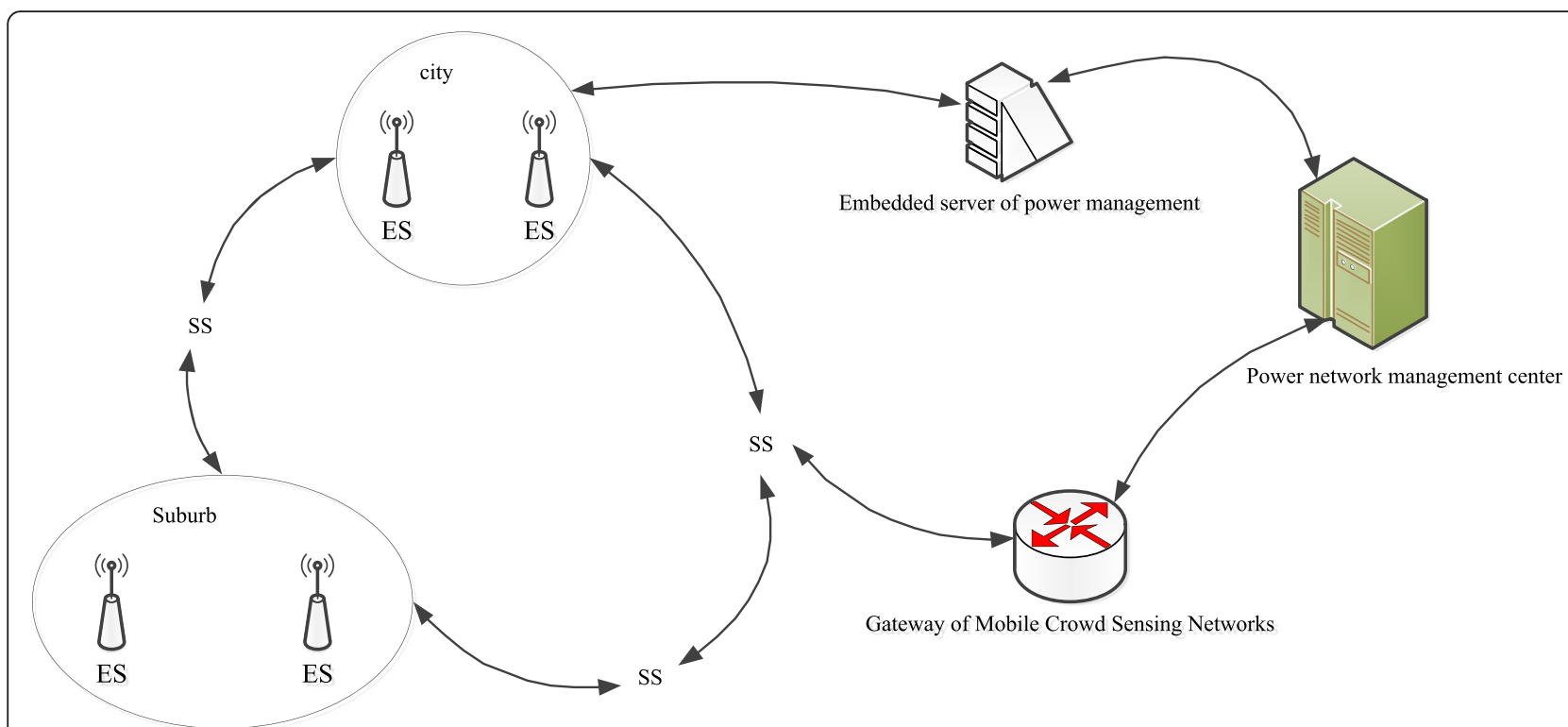

Fig. 1 The end-to-end communication between mobile crowd sensing networks and the embedded devices were established with the above four steps. Moreover, the mobile crowd sensing networks could provide the following grid data management functions for the embedded devices.

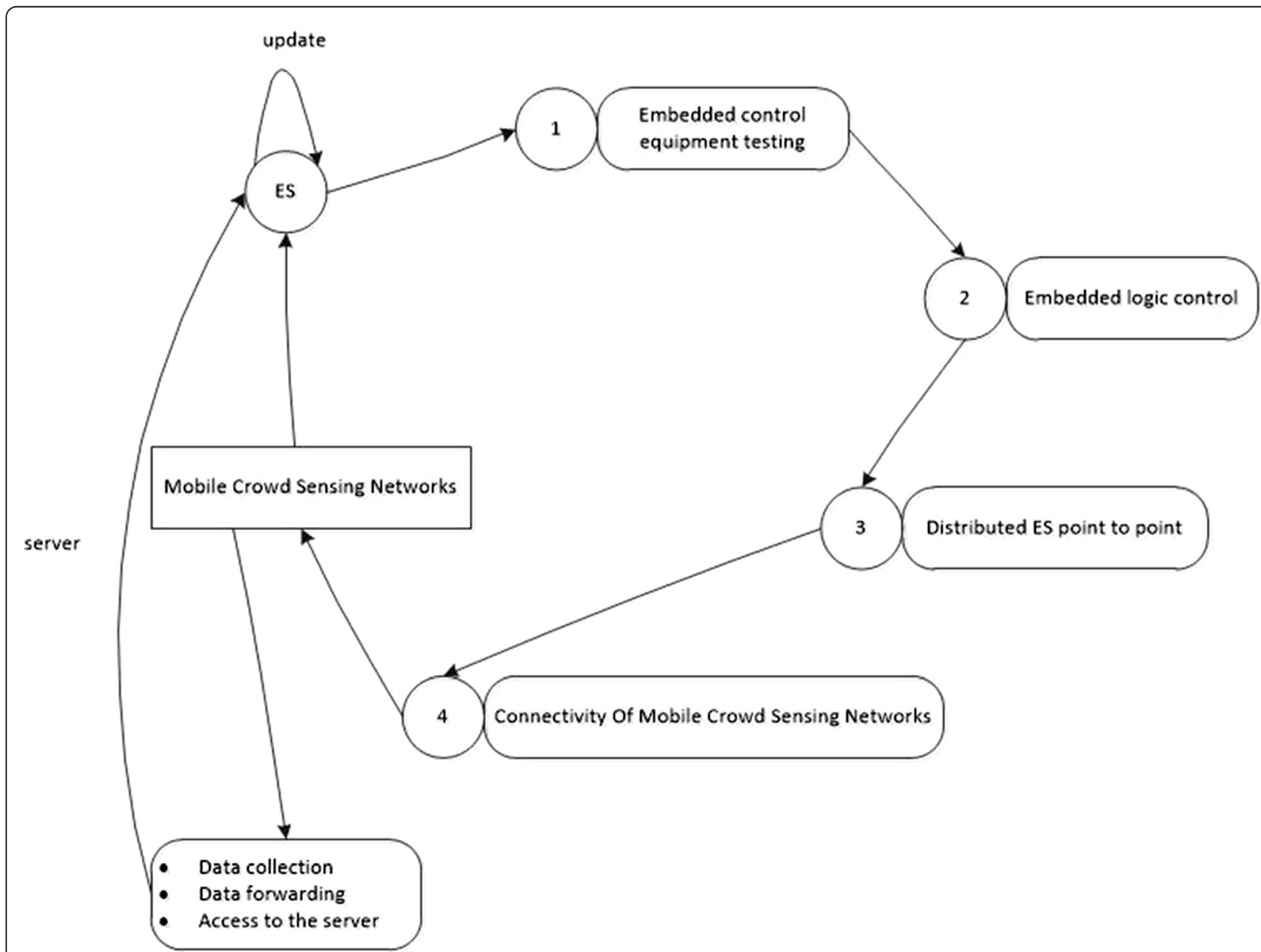

Fig. 2 Function platform of mobile crowd sensing embedded system 
(2)Data forwarding. Embedded devices have certain storage space, can store the data, and make sure the receiver forwards the data after creating an end-to-end communication link.

(3) Access to the server. A communication link between power grid management servers was established for helping the embedded devices to gain the access permissions of the servers.

The urban grid management would be researched after the deployment of mobile crowd sensing network. Assumptions embedded devices with functions of perception, collection, and data forwarding. At the same time, each embedded devices has mobile ability, and in accordance with the established time sequence point moves, has a fixed mobile trajectory. Mobile embedded devices positioning can be achieved by time and speed.

In a time domain to a known period, analysis for the power grid management of mobile crowd sensing network coverage quality and work with mobile embedded devices combined with cycle and activity were done.

In a spatial domain, combined with the feature of urban buildings and all kinds of noise source, on the basis of time domain integral, the mobile embedded devices perceive an orthogonal segment and can, according to the power grid management, control center service application requests and intelligent decision power grid management level of precision and reliability.

Therefore, in each round of data collection, store, and forward cycle, to meet the power grid management service request at the same time, combined with the feature of coverage, distributed embedded cover, covering the size as shown in formula (1), if formula (2) is met, shows the realizing of a complete coverage of the area.

$$
P_{\mathrm{S}}(\mathrm{SS})=\left\{\begin{aligned}
1, t & <\frac{N_{\mathrm{T}}}{N_{\mathrm{ES}}} \\
0, t & =\frac{N_{\mathrm{T}}}{N_{\mathrm{ES}}} \\
\frac{t}{T}, t & >\frac{N_{\mathrm{T}}}{N_{\mathrm{ES}}}
\end{aligned}\right.
$$

Here, $P_{\mathrm{S}}$ denotes the SS cover granularity. $N_{\mathrm{T}}$ presents the monitored time length. $N_{\mathrm{ES}}$ denotes the mobile embedded devices. $T$ is the delay of the data collection, store, and forward cycle.

$$
\sum_{i=1}^{\frac{T}{t}} P_{\mathrm{S}}\left(\mathrm{SS}_{\mathrm{i}}\right) \sum_{j}^{N_{\mathrm{ES}}} P_{\mathrm{SS}_{\mathrm{j}}}
$$

Here, $P_{S S j}$ denotes the connected probability.

\section{Mobile service aware opportunistic embedded system}

According to the requirements of intelligent power grid management, the deployment of the mobile group of crowd sensing network was studied. For embedded mobile front-end structures, highly integrated and two-way communication network at a high speed. In order to provide reliable, safe, economic, efficient, and environmental friendly management objectives and meet the power grid management mobile service application, mobile embedded system involves the following techniques: perception, sensor technology, data logic control technology, and independent decisions.

The system has the following characteristics:

(1) The embedded devices working state self-inspection Embedded device working condition is defined as

$\mathrm{EQS}=\left\{W_{\mathrm{v}}, P_{\mathrm{c}}, S_{\mathrm{p}}, F_{\mathrm{q}}, I_{\mathrm{g}}\right\}$

Here, $W_{\mathrm{v}}$ denotes the working voltage. $P_{\mathrm{c}}$ denotes the perceptual cycle. $S_{\mathrm{p}}$ denotes the storage space. $F_{\mathrm{q}}$ denotes the forward frequency. $I_{\mathrm{g}}$ denotes integration.

(2)Incentives for embedded devices.

In the mobile crowd sensing network, cloud storage, data sharing, and data grid quality evaluation of two-way link between the multiple embedded devices should be inspired with the multiple mobile embedded equipment state of common sense and mobile service requirements, therefore, to establish, as shown in formula (3), the incentive factors. Through these factors, to select the current mobile embedded devices, the best neighbor node subnet finishes the work.

$\left\{\begin{array}{l}M_{\mathrm{F}}=\sum_{i=1}^{N} \mathrm{EQS}_{i} \sqrt{\mathrm{AW}_{i}+\mathrm{MS}_{i}} \\ \sum_{i=1}^{N} \mathrm{AW}_{i} \geq \sum_{i=1}^{N} \mathrm{MS}_{i}\end{array}\right.$

Here, $N$ denotes the mobile devices scale of the mobile crowd sensing networks. $M_{\mathrm{F}}$ denotes the incentive factors. AW denotes the awareness ability. MS denotes the mobile service ability. Formula (3) shows that only when multiple embedded mobile devices of total demand, perception ability stronger than the mobile service to be effective incentive neighbor devices of mobile group of subnet.

(3) The mobile user requirements

Mobile user demand is equivalent to MS. Specific demand indicators include the following: embedded device control complexity, computational complexity, equipment capacity data storage space complexity, power supply feedback response delay, etc. 
(4) The anti-damage ability

Embedded mobile devices for neighbor device noise, noise of large power equipment, its own internal noise, bad environment embedded mobile communication reliable guarantee, etc.

(5) Mobile service awareness ability

The feature description is similar to the one in AW.

(6) Service awareness of the power quality guarantee Based on (3), (4) and (5), a comprehensive evaluation analysis of power management system would be given for providing power quality guarantee as the goal, by the formula (4), which is used to analyze the current mobile power quality guarantee ability.

$$
\left\{\begin{array}{l}
\mathrm{PQG}=\sum_{i=1}^{N_{\mathrm{SS}}} a_{i} \mathrm{AW}_{i}+b_{i} \mathrm{MS}_{i}+c_{i} A d_{i} \\
a_{i}+b_{i}+c_{i}=1, i \in\left[1, N_{\mathrm{SS}}\right] \\
\sum_{i=1}^{N} \mathrm{PQG}_{i} \geq \sum_{i=1}^{N} \mathrm{AW}_{i}
\end{array}\right.
$$

Here, $N_{\mathrm{SS}}$ denotes the subnet of the mobile devices.

(7)Diversity of power supply.

Embedded mobile devices compatible with all kinds

of power supply. The device to run in parallel with a single power supply or more class power has serial or parallel processing ability and has the comprehensive distributed power supply interface.

(8) Mobile power optimization

According to the mobile group of crowd sensing network communication load and speed embedded mobile devices, integrated optimization on the performance and the mobile service demand.

In order to achieve the above eight aspects of embedded devices and the characteristics of the communication control network, the need to design mobile service perceived opportunistic embedded to architecture, as shown in Fig. 3. The opportunity to architecture can be divided into two subsystems.

(1) The intelligent management of fault perception and self-healing smart grid system is suitable for high voltage distribution network. Through self-inspection of embedded mobile devices of short circuit and earth fault statistics, and the fault information to share with neighbors mobile equipment, working state is obtained by formula (3) value, thus for grid management provides the basis for transformer monitoring network and intelligent operation. At the same time, can be embedded mobile devices with two ways of connecting rod set up the intelligent electric opening and closing operation decision-making basis is given. The child system can obtain real-time current, voltage of the circuit changes in real time.

(2) The mobile group of the seamless combination of the net each subnet system. By moving the group of wisdom subnet to a large area power grid management area for unit division and the seamless connection, the system can state monitor real time the transmission lines and the grounding of the transformer equipment and subnet bolt bearing load, subnet, etc.

\section{Grid intelligent management of embedded systems}

Based on the above research work, through the mobile service awareness and opportunistic embedded system, we studied the suitable embedded mobile devices and proposed the power grid management based on mobile crowd sensing network management system, as shown in Fig. 4.

There are point-to-point communication between mobile service aware server, substation management side, and base station. The base station manages the mobile users and mobile crowd sensing gateways. The seamless coverage overlapping management and continuous repair and guarantee system would be used to guarantee the reliable service of submobile crowd sensing networks.

Assuming that the system is a continuous system. $y(t)=T\{\mathrm{EQ}(t) U(t)\}$. Here, $T$ presents the working hours of the power grid management system. Combined with embedded devices status, the power status of the continuous variation process of electric power was analyzed. When the system satisfies the conditions as shown in formula (5), the system has a linear characteristic.

$$
\left\{\begin{array}{l}
y_{1}(t)=T\{\mathrm{EQ}(t)\} \\
y_{2}(t)=T\{U(t)\} \\
y(t) \triangleright\left\|y_{1}(t)+y_{2}(t)\right\|
\end{array}\right.
$$

About the $v$ weighting parameters of every mobile service demand, the relationship of embedded system is shown in formula (6), which described the seamless coverage of the management features.

$$
\left\{\begin{array}{l}
|T\{v \mathrm{EQ}(t)\}-T\{v U(t)\}| \geq v y(t) \\
T\{v \mathrm{EQ}(t)\}=v T\{\mathrm{EQ}(t)\} \\
T\{v U(t)\}=v T\{U(t)\}
\end{array}\right.
$$

For adjacent mobile crowd sensing between the net $\mathrm{SS}_{1}$ and $\mathrm{SS}_{2}$, the overlap region, covering management through formula (7), is analyzed. 


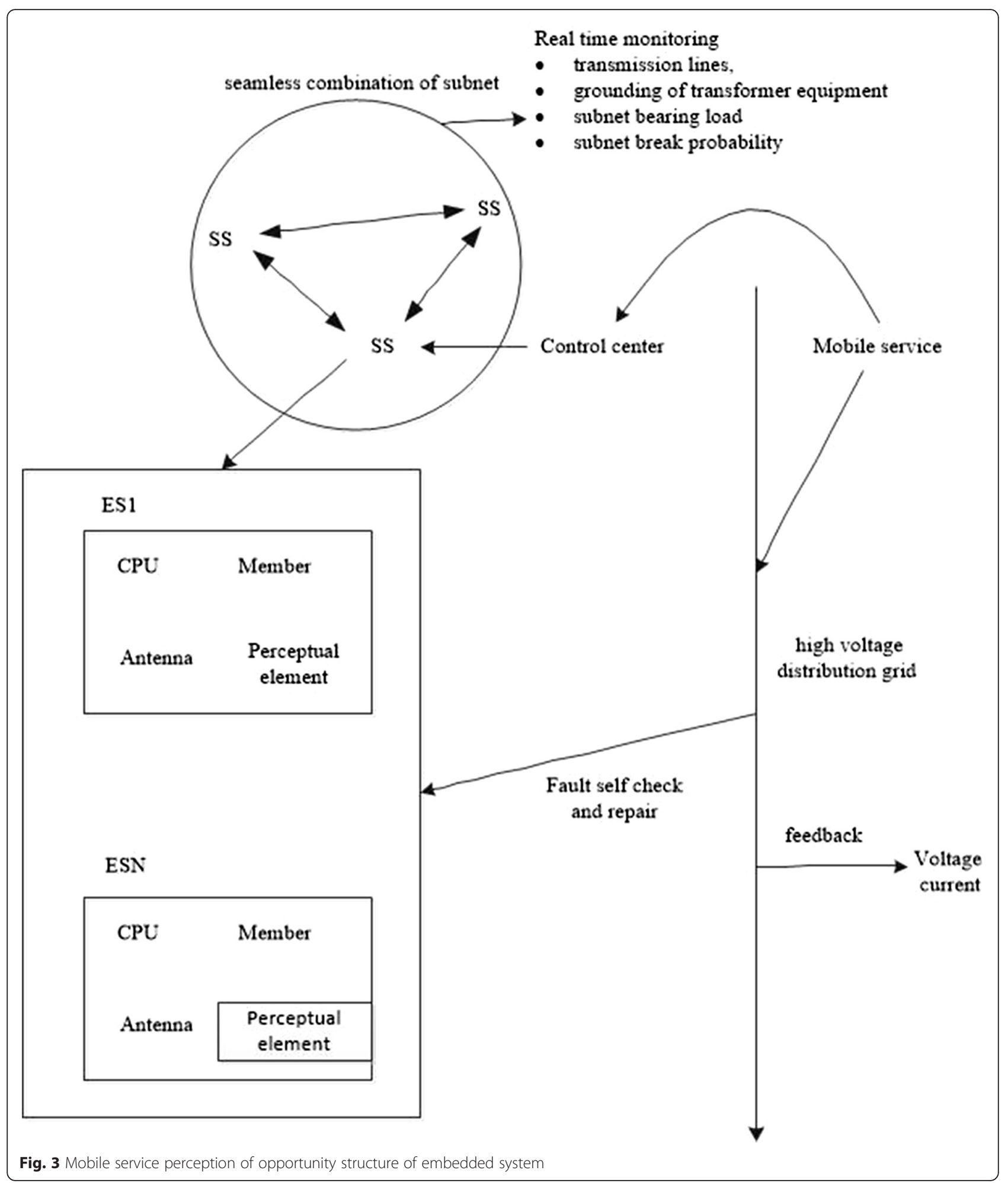




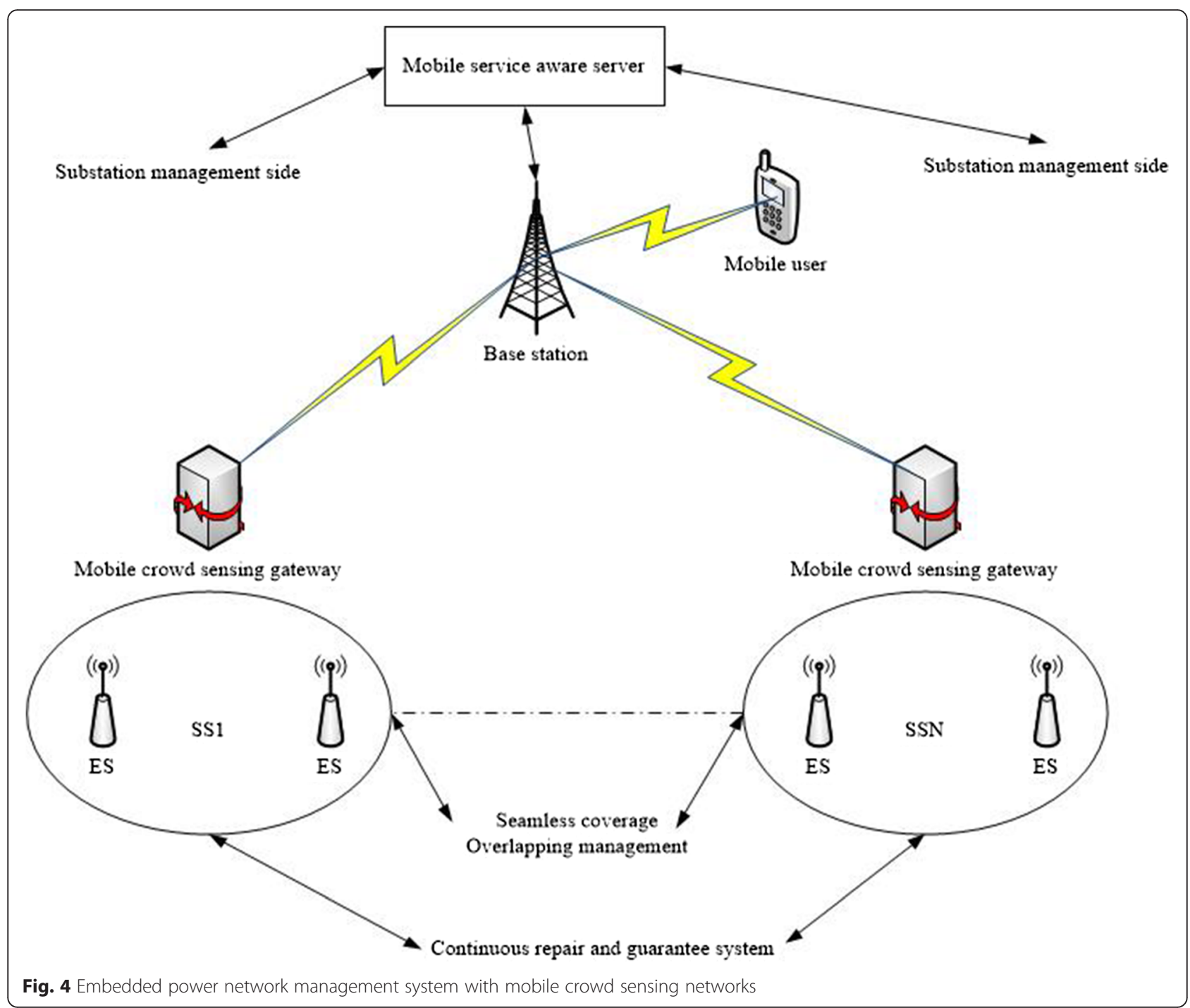

$$
\left\{\begin{array}{l}
T\left\{U_{\mathrm{SS}_{1}}(t)\right\}+T\left\{U_{\mathrm{SS}_{2}}(t)\right\} \approx \frac{\sqrt{T\{U(t)\}}}{N_{\mathrm{SS}}} \\
\mathrm{EQ}\left(\mathrm{SS}_{1}\right)>\frac{\sqrt{T\{\mathrm{EQ}(t)\}}}{N_{\mathrm{SS}}} \\
\mathrm{EQ}\left(\mathrm{SS}_{2}\right)>\frac{\sqrt{T\{\mathrm{EQ}(t)\}}}{N_{\mathrm{SS}}}
\end{array}\right.
$$

Therefore, the proposed embedded power management in the embedded system can well meet the practical engineering linear nuclear continuous electricity demand that continues to provide mobile users and diversity.

\section{The performance analysis of embedded system}

We form the following three aspects: the performance of the analysis and validation of the proposed embedded systems.

\subsection{The system reliability}

The power grid reliability of the mobile crowd sensing network intelligent embedded management system is that the power grid can provide stable power protection and mobile information services anytime and anywhere, at the same time, considering the quality of the embedded mobile devices, robustness and fault detection and repair ability. The performance can be obtained reference formula (8) analysis.

$$
N_{F}=\sum_{i=1}^{N} \sum_{j=1}^{N_{\mathrm{SS}}} j P\left(\mathrm{ES}_{i} \subset \mathrm{SS}_{i}\right)
$$

Here, $N_{\mathrm{F}}$ denotes the failure unit number per time. $P$ denotes the failure number of the mobile embedded devices in the mobile crowd sensing subnet networks. 


\subsection{The system manageability}

The management complexity considers computational complexity and control complexity of the integration of the software platform and hardware platform. In addition, the operation complexity and communication delay between the comprehensive evaluations of control center server, substation server, and embedded mobile devices would be studied in the meantime. The performance can be obtained reference formula (9).

$$
\mathrm{SC}=\sum_{i=1}^{N_{\mathrm{TRA}}} \alpha_{i} \sqrt{\mathrm{CPC}_{i}+\mathrm{CTC}_{i}}+\sum_{j=1}^{N_{\mathrm{SS}}} \beta_{j}\left|\mathrm{CO}_{j}-\mathrm{CL}_{j}\right|
$$

Here, SC denotes the complexity of the integrated system. $N_{\text {TRA }}$ denotes the transformer size. CPC denotes computational complexity. CTC denotes the control complexity. $\mathrm{CO}$ denotes the operation complexity. CL denotes the communication delays. $\alpha$ denotes the work frequency transformer. $\beta$ denotes the mobile group of the working time of the subnet.

\subsection{The system scalability}

On the basis of the diversity of user requirements and the dynamic expansion of mobile services, we would analyze the scalability based on the size of the mobile devices and the change of the coverage and the proposed system of power grid management performance, at the same time, for the embedded mobile equipment deployment way, communication protocol standards, as well as user operability and troubleshooting skills. The performance can be obtained by reference formula (10).

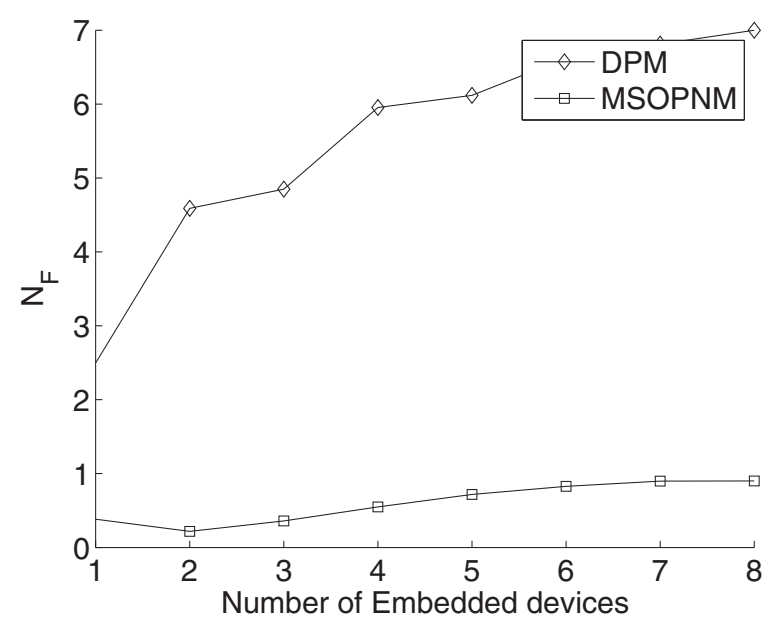

Fig. 5 Embedded system reliability

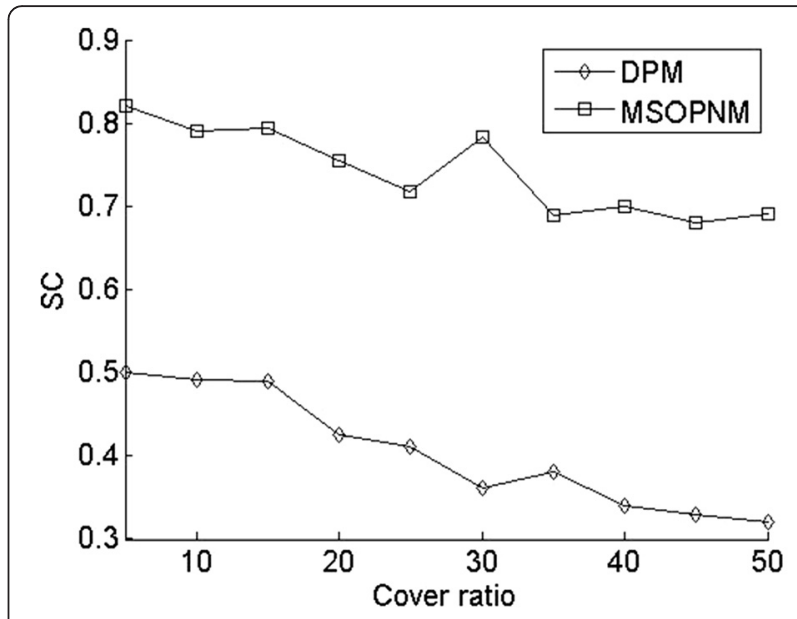

Fig. 6 Embedded system manageability

$$
\mathrm{SC}_{\mathrm{L}}=S_{\mathrm{C}} \frac{N_{\mathrm{TRA}} N_{\mathrm{SS}}}{N_{\mathrm{ES}}} P\left(P_{\mathrm{S}}-F_{\mathrm{c}}\right)^{\eta}
$$

Among them, the $\mathrm{SC}_{\mathrm{L}}$ denoted the system extension liquidate. $S_{\mathrm{C}}$ denoted the cover. $P_{\mathrm{S}}$ denotes the communication protocol standards available probability. $F_{\mathrm{C}}$ denotes mobile equipment failure repair probability. Said mobile service expansion factor.

The experimental environment description is as follows:

(1)Grid management scope: $10 \mathrm{~km} \times 12 \mathrm{~km}$

(2) Power grid management system running time: $24 \mathrm{~h}$

(3) Mobile crowd sensing subnet number: $2-5$

(4) Embedded mobile devices maximum communication distance of $1 \mathrm{~km}$

According to formulas (8), (9), and (10), the analysis of the mobile device number, percentage of coverage, and

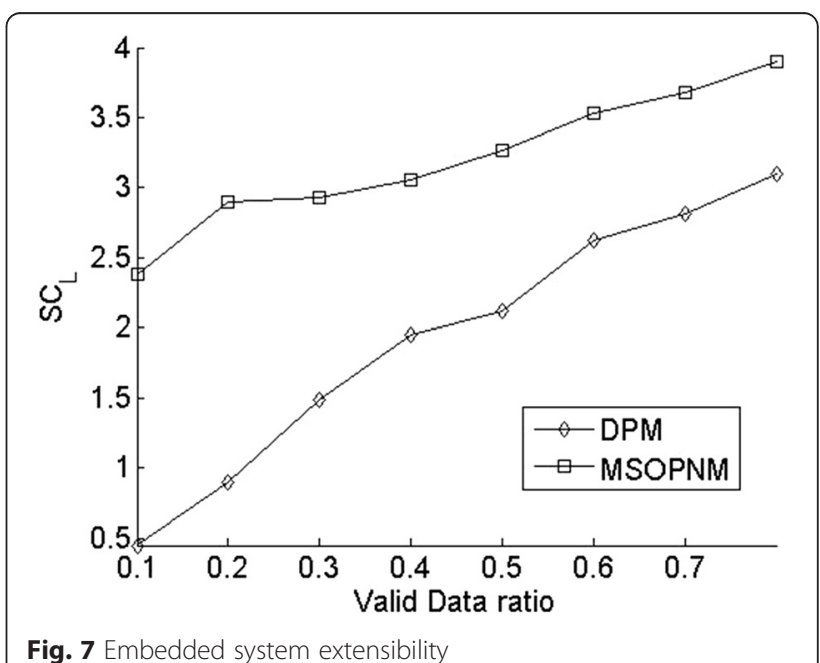


data size on the performance of the system reliability, manageability, and scalability, the proposed system (MSOPNM) with distributed power management (DPM) system performance is compared, and the results are shown in Figs. 5, 6, and 7.

Found from Fig. 5, the proposed system was built based on the mobile service awareness for mobile devices because of the high reliability of embedded system structure, while continuously increasing the scale of mobile devices but still keeping good performance, and is obviously better than the reliability of distributed network management system. For distributed network management system through a long response delay for mobile devices, the substation builds end-to-end communication server and, therefore, management performance, as shown in Fig. 6. Faced with massive data collection, store, and forward cycle, the proposed system can be used as user requirements for diversity and dynamic expansion of mobile services to provide effective guarantee, even if the cover dynamic change still has good performance of power grid management, as shown in Fig. 7.

\section{Conclusions}

The traditional power supply and power grid management schemes are unstable and inefficient for the rapid development of the human daily life and work. For dealing with the above issues, we researched the opportunistic embedded architecture for the power network measurement with the mobile service aware scheme and mobile crowd sensing networks. About the intelligent power grid management, we designed the mobile crowd sensing network for power grid management. Then, according to the requirement diversity of the intelligent power grid management and deployment of the mobile crowd sensing networks, the mobile service aware opportunistic embedded system was studied. Finally, we proposed the grid intelligent management scheme and system with embedded systems. The experimental results show that the proposed scheme is superior to distributed power management system, such as the system complexity, execution efficiency, intelligent power grid management level, etc.

\section{Competing interests}

The authors declare that they have no competing interests.

Received: 13 November 2015 Accepted: 19 January 2016 Published online: 03 February 2016

\section{References}

1. $\mathrm{BHe}, \mathrm{R}$ Nie, $\mathrm{K}$ Chu, Research on application of electric energy detection system based on intelligent power network management platform. J. Changzhou Univ. 23, 47-50 (2011). No.3

2. F Wang, W Gu, X Yuan et al., Power quality management platform for smart grid. Electric Pow. Automat. Equip. 32(7), 134-139 (2012)
3. Chao $L$, Shuang $L$, Shiming $W$. Integration framework of smart grid management services system. Jornal of Jln Nvry Ngnrng \& Hnology Don. 42 Sup.1, 246-250, 2012.

4. $\quad$ Y Huang, $X$ Zhou, Generalized data management model of smart grid based on ontology theory. Electric. Pow. Sys. Automat. 38, 9 (2014)

5. J Zhongyuan, Z Xiuyan, M Ziyue, Capacity optimization for power storage systems in smart grids. J. Xidian University 42(6), 124-130 (2015)

6. J Li, G Wu, Y Liu, Application of RUDP protocol in embedded data communication. Terminal Unit of Meteorological equipment 4, 45-48 (2013)

7. C You-qing, Y Shao-hua, Research on TCP high availability technology for embedded network device. J. Chin. Comp. Sys. 34(2), 199-204 (2013)

8. L Lua, LI Xiao-jina, Z Honga et al., Embedded and mobile device software low-power optimization methods based on storage system's accessing characteristics. Appl. Res. Comput. 31(11), 3392-3396 (2014)

9. R Yao, T Ya-guang, W Wei-feng, Design of a UV monitoring system based on embedded system for electric power equipments. Sensor World. 20(10), 21-25 (2014)

10. G Chen, F Zhang, C Xu, Embedded soft system for integrated circuit burn in test device. Comput. Digi. Eng. 42(5), 891-895 (2014)

11. C Xiang, X Jia, W Min. Research of data collection technology in crowd sensing based on social behavior analysis. Application Research of Computers. 2015;32(12):3534-41.

12. H Han Xia, D Qiang, L Li, Research on mobile terminal crowdsourcing. Comp. Technol. Dev. 24(6), 6-9 (2014)

13. J Zhang, B Guo, X Lu et al., Approach for urban popular event detection using mobile crowdsourced data. Comp. Sci. 42(6A), 5-9 (2015)

14. N Wenqian, G Bin, C Huihui. A Cross-Space, Multi-Interaction-Based Dynamic Incentive Mechanism for Mobile Crowd Sensing. Chinese J Comp. 2015; 38(12):2412-25.

15. R Pérez-Andradea, C Torres-Huitzila, R Cumplidob, Processor arrays generation for matrix algorithms used in embedded platforms implemented on FPGAs. Microprocess. Microsyst. 39(7), 576-588 (2015)

16. MA Murillo-Escobara, C Cruz-Hernándezb, F Abundiz-Péreza et al., A robust embedded biometric authentication system based on fingerprint and chaotic encryption. Expert Sys. Appl. 42(21), 8198-8211 (2015)

17. X Fang, PG Charalambides, The fracture mechanics of cantilever beams with an embedded sharp crack under end force loading. Eng. Frac. Mech. 149, 1-17 (2015)

18. Z Vladimir, T Mirko, K Ljupco, Dynamic intelligent load balancing in power distribution networks. Int. J. Electr. Power Energy Syst. 73, 157-162 (2015)

19. C Yul, P Jin Hwan, Dual-access way-prediction cache for embedded systems. EURASIP J. Embed. Syst. 2014, 16 (2014)

20. K Chutisant, A methodology for hand and finger motion analysis using adaptive probabilistic models. EURASIP J. Embed. Syst. 2014, 18 (2014)

\section{Submit your manuscript to a SpringerOpen ${ }^{\odot}$ journal and benefit from:}

- Convenient online submission

- Rigorous peer review

- Immediate publication on acceptance

- Open access: articles freely available online

- High visibility within the field

- Retaining the copyright to your article

Submit your next manuscript at $>$ springeropen.com 